\title{
Radionuclide Myocardial Perfusion Imaging for the Evaluation of Patients With Known or Suspected Coronary Artery Disease in the Era of Multimodality Cardiovascular Imaging
}

\author{
Viviany R. Taqueti and Marcelo F. Di Carli* \\ Noninvasive Cardiovascular Imaging Program, Departments of Medicine and Radiology, Division \\ of Nuclear Medicine and Molecular Imaging, Department of Radiology, Cardiovascular Division, \\ Department of Medicine, Brigham and Women's Hospital, Harvard Medical School, Boston, MA
}

\begin{abstract}
Over the last several decades, radionuclide myocardial perfusion imaging (MPI) with single photon emission tomography and positron emission tomography has been a mainstay for the evaluation of patients with known or suspected coronary artery disease (CAD). More recently, technical advances in separate and complementary imaging modalities including coronary computed tomography angiography, computed tomography perfusion, cardiac magnetic resonance imaging, and contrast stress echocardiography have expanded the toolbox of diagnostic testing for cardiac patients. While the growth of available technologies has heralded an exciting era of multimodality cardiovascular imaging, coordinated and dispassionate utilization of these techniques is needed to implement the right test for the right patient at the right time, a promise of "precision medicine." In this article, we review the maturing role of MPI in the current era of multimodality cardiovascular imaging, particularly in the context of recent advances in myocardial blood flow quantitation, and as applied to the evaluation of patients with known or suspected CAD.
\end{abstract}

\section{Keywords}

Multimodality cardiovascular imaging; Radionuclide myocardial perfusion imaging; Ischemic heart disease; Positron emission tomography; Coronary flow reserve

\begin{abstract}
Over the last several decades, radionuclide myocardial perfusion imaging (MPI) with single photon emission tomography (SPECT) and positron emission tomography (PET) has been a mainstay for the evaluation of patients with known or suspected coronary artery disease (CAD). ${ }^{1}$ More recently, technical advances in separate and complementary imaging modalities including coronary computed tomography angiography (CCTA), computed tomography perfusion (CTP), cardiac magnetic resonance imaging (CMR), and contrast
\end{abstract}

\footnotetext{
*Address reprint requests to: Marcelo F. Di Carli, MD, Brigham and Women's Hospital, ASB-L1, Room 037 C, 75 Francis St, Boston, MA 02115. mdicarli@ partners.org (M.F. Di Carli).

Statement of Conflict of Interest: None.

Disclosures

None.
} 
stress echocardiography (SE) have expanded the toolbox of diagnostic testing for patients evaluated for cardiovascular (CV) diseases. While the growth of available technologies has heralded an exciting era of multimodality $\mathrm{CV}$ imaging, coordinated and dispassionate utilization of these techniques is needed to implement the right test for the right patient at the right time, a promise of "precision medicine." 2 This article will review the maturing role of MPI in the current era of multimodality CV imaging, particularly in the context of recent advances in myocardial blood flow (MBF) quantitation, and as applied to the evaluation of patients with known or suspected CAD.

\section{Multimodality appropriate use}

At its peak nearly a decade ago, an estimated 40 million noninvasive cardiac examinations were beingperformed annually in the United States, with cardiac imaging accounting for a considerable proportion of the Medicare budget. ${ }^{3,4}$ Recognizing the relative explosion of new cardiac imaging technologies and its impact on the practicing clinician, the American College of Cardiology along with other professional societies recently published the first Multimodality Appropriate Use Criteria, in which stress tests and anatomic diagnostic procedures are rated side-by-side for the same indication in the assessment of patients with stable CAD. ${ }^{5}$ Because of limited availability of comparative data across different modalities, as well as variability in patient populations and test availability at any specific local setting, these ratings were deliberately noncompetitive.

\section{Strengths and limitations of radionuclide MPI}

Radionuclide MPI effectively began in the early 1970s with the first reports of noninvasive evaluation of MBF at rest, and subsequently, with the addition of ${ }^{201} \mathrm{Tl}$ MPI to electrocardiogram (ECG) exercise treadmill (ETT) testing. ${ }^{6}$ Since then, significant technological advances in hardware, software, and radiopharmaceuticals have transformed the field of nuclear cardiology (Fig 1). Currently, imaging techniques such as SPECT and PET allow for the assessment of flow-limiting CAD by evaluation of myocardial perfusion, metabolism, and also left ventricular (LV) contractile function. A wealth of data has established the role of contemporary radionuclide MPI in both the diagnosis and management, as well as the prognosis of patients being evaluated for known or suspected CAD.

\section{Diagnostic accuracy}

The role of any imaging procedure is to provide information to improve the clinician's decision-making process with the ultimate goal of ameliorating patient symptoms and/or bettering clinical outcomes. As such, clinical interpretation of radionuclide MPI studies relies upon sequential Bayesian analysis of disease probability, where the post-test probability of disease is influenced not only by the sensitivity and specificity of the test, but also by the pretest probability of disease. In the setting of chest pain, the prevalence or pretest probability of CAD varies depending on patient characteristics including age, gender, and coronary risk factors, as well as the nature of the presenting symptoms. In this way, patients with very low or high pretest probabilities of CAD are unlikely to benefit from the addition of noninvasive imaging for the diagnosis of CAD or cardiovascular risk 
stratification, regardless of the imaging modality used. ${ }^{7}$ Furthermore, with any imaging modality, diagnostic accuracy is limited by post-test referral bias, which inflates the sensitivity and deflates the specificity of the test. ${ }^{8}$

For those patients with truly intermediate pretest probabilities of disease, noninvasive imaging has a well-established role in the diagnosis of CAD. ${ }^{5}$ Radionuclide MPI with SPECT using either exercise or pharmacologic stress testing remains the most common form of stress imaging in the evaluation of patients with known or suspected CAD, and represents a robust approach for diagnosing flow-limiting (i.e., obstructive) CAD. Early published literature (based on a pooled analysis of 79 studies of nearly 9000 patients) with SPECT reported an average sensitivity of $86 \%$ and an average specificity of $74 \%$ for detecting $>50 \%$ angiographic stenosis, ${ }^{9}$ with improved specificity using attenuation correction methods. ${ }^{10}$ With PET perfusion imaging, the reported average sensitivity was $90 \%$ and the average specificity was $89 \%$ for detecting $>50 \%$ angiographic stenosis, as derived from a pooled analysis of 9 studies of almost 900 patients. ${ }^{11}$ A recent meta-analysis of nearly 12,000 patients in 177 studies (108 SPECT MPI, 4 PET MPI, and 5 PET and SPECT MPI) confirmed higher sensitivity of PET relative to SPECT MPI (92.6\% vs. 88.3\%, $p=0.035)$ for detection of $>50 \%$ epicardial stenosis, with comparable specificity reported between techniques $(81.3 \%$ vs. $75.8 \%, p=0.39)$ in the pooled analysis. ${ }^{12}$ Similar findings were reported in a smaller meta-analysis, which showed incremental improvement in accuracy with PET relative to SPECT for the diagnosis of obstructive CAD (area under the receiveroperating characteristic curve of 0.95 and 0.90 for PET and SPECT, respectively, $p<$ $0.0001) .{ }^{13}$

Several technical advantages account for the improved image quality and diagnostic ability of PET compared to SPECT including: (1) routine measured (depth-independent) attenuation correction, which decreases false positives and thereby increases specificity; (2) high spatial and contrast resolution (heart-to-background ratio), which allows improved detection of small perfusion defects, thereby decreasing false negative and increasing sensitivity; and (3) high temporal resolution, which allows fast dynamic imaging of tracer kinetics and makes possible absolute quantification of myocardial perfusion (in $\mathrm{mL} / \mathrm{min} / \mathrm{g}$ of tissue). ${ }^{14}$

A multitude of studies in the cardiovascular imaging literature have reported on the relative diagnostic accuracies of the various imaging modalities, with slight variations in results depending on specific disease prevalence of the clinical cohorts evaluated. Conceptually, the relative sensitivities and specificities of these imaging techniques, from radionuclide MPI with SPECT or PET to stress echocardiography (SE), stress CMR (with or without coronary MR angiography, CMRA), and CCTA, can be understood as a function of the pathophysiologic ischemic cascade ${ }^{15}$ (Fig 2A). Even prior to the onset of symptomatic ischemia is the development of subclinical CAD, which may be readily detectable as atherosclerotic plaque by CCTA. Ensuing progressive endothelial and microvascular dysfunction may not be detectable by radionuclide MPI until it is associated with maldistribution of MBF and frank coronary hypoperfusion [unless PET-based quantitation of MBF and coronary flow reserve (CFR) is also performed]. Defects in myocardial perfusion may progress to cardiomyocyte dysfunction, involving both impaired diastolic relaxation 
and systolic regional wall motion abnormalities, which may become evident on SE. Enough ischemia and impaired cardiomyocyte mechanics may ultimately lead to gross electrocardiographic abnormalities and reproducible symptoms on ETT alone. It is therefore not surprising that the anatomic assessments of atherosclerotic plaque (i.e., CCTA, CMRA) demonstrate increased sensitivity (and decreased specificity), whereas the increasingly functional techniques (i.e., SE) demonstrate increased specificity (and decreased sensitivity) for the diagnosis of CAD relative to radionuclide MPI. This reflects the importance of a multimodality approach for interrogation of CAD along the spectrum of disease (Fig 2B).

After SPECT MPI, SE is the next most commonly utilized noninvasive imaging technique for the diagnosis of obstructive CAD. Although the use of IV contrast agents may eventually allow for the assessment of myocardial perfusion, the majority of current SE studies rely on the induction of regional wall motion abnormalities and reduced systolic wall thickening for the detection of myocardial ischemia. Pooled analysis of 43 studies on the diagnostic accuracy of SE showed an average sensitivity of $84 \%$ and specificity of $82 \%$ for exercise SE, compared with $80 \%$ and $84 \%$, respectively, for dobutamine SE. ${ }^{9}$ Direct comparisons between SE and SPECT with different stressors (including both exercise and pharmacologic vasodilators) revealed an incrementally higher overall sensitivity for SPECT MPI as compared to SE ( $84 \%$ vs. $80 \%, p=0.029$ ), with higher specificity for SE as compared to SPECT MPI ( $86 \%$ vs. $77 \%, p=0.001) .{ }^{16}$

Stress CMR imaging combines both the evaluation of regional myocardial perfusion and inducible regional wall motion using pharmacologic stress agents. For myocardial perfusion, pooled analysis of 17 studies in over 500 patients yielded an average sensitivity of $84 \%$ and specificity of $85 \%$ for the detection of CAD. ${ }^{9}$ For wall motion, pooled analysis of 10 studies including over 600 patients reported an average sensitivity of $89 \%$ and $84 \% .{ }^{9}$ Although the addition of wall motion to myocardial perfusion analysis in response to dobutamine stress improves the sensitivity for the diagnosis of $\mathrm{CAD}$, this may not increase the overall diagnostic accuracy because of a concomitant decrease in specificity. ${ }^{17}$

Although functional assessment of ischemia provides a reliable evaluation of patients with known or suspected CAD, relatively recent advances in noninvasive imaging of coronary anatomy, particularly using CCTA, have generated interest in the detection of atherosclerosis itself. A summary of published studies investigating the diagnostic accuracy of CCTA has reported the sensitivity and specificity per patient to be $94 \%-97 \%$ and $83 \%-90 \%$, respectively. ${ }^{18}$ Although CCTA can reliably exclude obstructive CAD with per-vessel negative predictive values of $99 \%,{ }^{19}$ the reported positive predictive value is much lower and highly dependent on patient selection. The prospective multicenter ACCURACY trial reported a sensitivity of $93.8 \%$ and a specificity of $81.8 \%$ for detecting a coronary stenosis $>70 \%$, with misidentification of stenosis severity in more than half of the cases. ${ }^{19}$ Similarly, less than half of patients with a CCTA-diagnosed stenosis severity of $>50 \%$ demonstrate ischemia detectable by SPECT or PET. ${ }^{20,21}$ These findings highlight the highly variable relationship between anatomic stenosis severity and myocardial ischemia.

Indeed, the traditional use of coronary angiography as a gold standard for noninvasive cardiac imaging is problematic because anatomic CAD is neither necessary nor sufficient for 
the presence of myocardial ischemia and prediction of benefit from revascularization. This was further illustrated in a prospective study of 752 patients directly comparing CMR (perfusion, wall motion, and coronary angiography) and SPECT MPI for evaluation of suspected angina, which reported similar specificity but dramatically decreased sensitivity for SPECT MPI relative to CMR in the detection of angiographically-significant stenosis on subsequent invasive angiography (sensitivity of SPECT and CMR 66.5\% vs. 86.5\%, respectively, $p<0.0001$, and specificity was $82.6 \%$ vs. $83.4 \%$, respectively, $p=0.92$ ). The significantly lower sensitivity demonstrated for SPECT MPI in this prospective study (in which all patients underwent subsequent invasive $\mathrm{x}$-ray angiography regardless of imaging finding and only $11 \%$ of patients had previous revascularization or acute coronary syndrome) underscores the tension between an anatomic versus functional approach for the detection of clinically significant CAD. As such, radionuclide MPI (and more recently, PETbased quantification of MBF and CFR) provides an important functional assessment of ischemic burden, linking coronary anatomy to prognosis in patients spanning the full anatomic spectrum of CAD.

\section{Prognostic value}

For decades, radionuclide MPI has provided incremental value for risk assessment in patients with known or suspected CAD. There is a linear risk of cardiac death and myocardial infarction (MI) associated with increasing extent and severity of perfusion abnormalities. ${ }^{22,23}$ As with SE, stress MPI allows further clinical risk stratification, or incremental prognostic value, even after stratifying for pre-imaging data ${ }^{24}$ (Fig 3). In contrast to less sensitive imaging techniques, a normal or low-risk SPECT study is associated with a low major adverse $\mathrm{CV}$ event rate of $0.6 \%$ per year, as reported in a metaanalysis of 19 studies of nearly 40,000 patients evaluated over ten years, ${ }^{25}$ and comparable to event rates in the general population without evidence of CAD. Similar findings have been demonstrated for PET MPI, where a normal scan indicates low risk $(<1 \%$ annual CV event rate) while an abnormal scan indicates worse prognosis ( $>4.2 \%$ annual event rate). ${ }^{14}$ Furthermore, the integration of perfusion and function, enabled by seamless assessment of LV ejection fraction and volumes with radionuclide MPI, provides key prognostic information. In an early study of 2686 patients followed over a mean of nearly two years, the magnitude of perfusion defect reversibility, after adjustment for clinical factors, remained the strongest predictor of nonfatal MI, whereas cardiac death was best predicted by poststress LV ejection fraction. ${ }^{26}$ This observation highlights that, whereas the degree of myocardial ischemia may be a marker for future coronary events, $\mathrm{CV}$ death may be better predicted by myocardial dysfunction and scar. Even in the presence of angiographically documented CAD, normal findings on stress MPI are generally associated with a low risk of $\mathrm{CV}$ events at approximately $1 \%$ per year. ${ }^{25}$

Nonetheless, in contrast to CCTA, radionuclide MPI may identify only coronary territories supplied by the most severe stenosis, and therefore may be a relatively insensitive technique to delineate the full extent of cardiac atherosclerotic burden, especially in the setting of multivessel disease. This is an area where PET MPI holds great potential. In addition to the possibility of assessing the delta change in LV ejection fraction from baseline to peak stress (with the use of a short half-life tracer such as rubidium-82), there is diagnostic and 
prognostic power in the quantitation of MBF and CFR as permitted by PET MPI. In fact, CFR, calculated as the ratio of hyperemic to rest absolute MBF, is an integrated measure of coronary vasomotor dysfunction that integrates the hemodynamic effects of focal, diffuse, and small-vessel CAD on myocardial tissue perfusion, ${ }^{27}$ and is emerging as a noninvasive, quantitative prognostic imaging marker of $\mathrm{CV}$ risk. The accuracy of quantitative noninvasive PET measures of MBF and CFR by PET has been extensively validated in experimental animals and humans, and the reproducibility of this technique is well-established. ${ }^{28,29}$ PET is considered the gold standard for quantifying MBF and CFR. Growing data have consistently shown that CFR measurements by PET can distinguish patients at low or high risk for serious adverse events, including $\mathrm{CV}$ death, ${ }^{30-33}$ beyond comprehensive clinical assessment, LV ejection fraction, or traditional semi-quantitative measures of stress-induced ischemia $^{30}$ (Fig 4).

Addition of CFR, as measured by PET, provides incremental risk stratification that leads to significant and meaningful risk-reclassification (of approximately 35\%) of intermediaterisk patients. ${ }^{30-33}$ A preserved CFR (>1.9) effectively excluded high-risk angiographic CAD with a sensitivity of $86 \%$ and a negative predictive value of $97 \%$ in patients with normal or mildly to moderately abnormal ( $<10 \%$ LV ischemia and/or scar) PET MPI. ${ }^{34}$ Conversely, a CFR of $<2$ has been associated with a 3.4-fold increased hazard of cardiac death, and a CFR $<1.5$ has been associated with a 5.6-fold increased risk of cardiac death. ${ }^{30}$ Although global CFR is only modestly associated with the overall extent and severity of angiographic disease, both low CFR and high angiographic disease score were independently associated with adverse clinical events ${ }^{35}$ (Fig 5). In addition, global CFR modified the effect of revascularization such that only patients with low CFR appeared to benefit from revascularization, and only if the revascularization included coronary artery bypass grafting. 35 The finding that global CFR is associated with major CV events independently of luminal angiographic severity and modifies the effect of coronary revascularization underscores the morbidity associated with diffuse atherosclerosis and microvascular disease.

This has been illustrated in diabetic patients, who demonstrate impaired coronary vasoreactivity even in the absence of obstructive atherosclerosis ${ }^{36}$ and in whom absence of myocardial ischemia on noninvasive testing does not necessarily identify a lower-risk cohort. 25,37 In contrast to diabetic patients without known CAD with preserved CFR (who demonstrate very low levels of risk), diabetic patients without known CAD with impaired CFR showed a risk of $\mathrm{CV}$ death comparable to, and possibly higher than, that for nondiabetic patients with known CAD (2.8\% vs. $2.0 \%, p=0.33)^{37}$ (Fig 6). These observations suggest that impaired CFR may be a more powerful biomarker for diffuse atherosclerosis than diabetes mellitus alone. Indeed, even in patients presenting with chest pain or dyspnea with an intermediate pretest probability of CAD and in whom significant disease was ruled out with conventional clinical diagnostics and normal PET MPI, an impaired global CFR $(<2)$ was independently associated with a minimally elevated serum cardiac troponin and major adverse $\mathrm{CV}$ events. ${ }^{38}$ Impaired CFR, here reflecting microvascular dysfunction, modified the effect of a positive troponin on adverse outcomes such that only troponin-positive patients with concomitant impairment in CFR experienced worse cardiovascular outcomes in this otherwise low risk population. 
These findings support that global atherosclerotic disease burden and resultant macro- and microvascular ischemia, with or without obstructive epicardial lesions, are important contributors to overall CV risk, especially when hard outcomes such as CV death are considered. As such, PET-derived CFR may have particular prognostic importance as a sensitive and global biomarker for functional CAD, especially in its ability to integrate complex pathophysiologic sequelae at the target organ of interest. Consistent with this concept, nonobstructive and obstructive CAD, as defined by CCTA, are associated with similar risks of $\mathrm{MI}$ and death if the extent and severity of atherosclerotic plaques affect a larger number of arterial segments. ${ }^{39}$

A hybrid approach of combined anatomical and perfusion-based imaging is appealing. For patients with low-intermediate pretest probability, CCTA with its high negative predictive value may be an appropriate choice. For patients with intermediate and intermediate-high pretest probability, as well as special populations including diabetics and the elderly (in whom risk is likely to be heterogeneous), PET/CT with or without CFR quantitation, may add substantial diagnostic and prognostic value. As reviewed above, despite an excellent negative predictive value, CCTA is a poor discriminator of patients with myocardial ischemia. Conversely, a normal stress PET study is a poor discriminator of patients without evidence of non-flow-limiting (subclinical) coronary atherosclerosis. These findings support potentially complementary roles of anatomic and perfusion imaging in the evaluation of patients with suspected $\mathrm{CAD}^{20}$ (Fig 2).

Ultimately, the idea of using prognosis as a measure of added value from imaging is critically important but difficult to achieve. Because prognosis is closely tied to management, the use of improved health outcomes as a critical way to measure quality and value is limited by the inevitable heterogeneity of downstream clinical intervention triggered by the imaging test (which may effectively uncouple the clinical outcome from the imaging result). This concept has been illustrated in prospective registries such as the Study of Perfusion and Anatomy's Role in CAD (SPARC), which showed that noninvasive testing had only a modest impact on clinical management of patients referred for clinical testing. Among patients with the most severe test result findings, up to $61 \%$ were not referred to diagnostic coronary catheterization and $20 \%-25 \%$ were not receiving a lipid-lowering agent at 90 days after index imaging. ${ }^{40}$

\section{Other clinical considerations: radiation, throughput, availability, and cost}

Unlike SE or CMR, radionuclide MPI, unavoidably, involves radiation. However, carefully applied basic principles coupled with thoughtful clinical protocols, such as the introduction of stress-only imaging ${ }^{41}$ when feasible and the near elimination of dual-isotope studies, have allowed for a decrease in radiation dosage associated with mainstream SPECT MPI. PET MPI, with the use of short-lived radiopharmaceuticals, has the added advantage of much lower radiation doses, as well as fast, sequential assessment of regional myocardial perfusion at rest and stress, ${ }^{14}$ thereby improving laboratory efficiency and patient throughput. In contrast to echocardiography and CMRI, radionuclide MPI is not as highly operator-dependent, and with advances in hardware and software, less limited by patient body habitus and motion. Despite these technical advantages, access to PET for routine MPI 
remains somewhat limited. Currently, approximately 200 medical centers in the US, as well as several medical centers in Canada and Europe offer clinical PET MPI services. ${ }^{14,42} \mathrm{~A}$ promising new radiotracer, flurpiridaz F-18, which exhibits superior imaging quality and does not require an on-site cyclotron, may significantly expand PET for MPI ${ }^{42}$ Flurpiridaz F-18 combines high resolution enabled by a short positron range, with high sensitivity to detect milder perfusion defects and accurately quantify MBF, as enabled by near linear myocardial extraction across the physiologic range of $\mathrm{MBF}^{43}$ If eventually approved by the US Food and Drug Administration and embraced by clinical payors, this novel radiotracer may one day allow PET MPI at any facility with existing (i.e., fluorodeoxyglucose, FDG) PET capability.

Finally, recent studies illustrate the challenges associated with accurate assessment of pretest risk and clinical decision-making in daily practice. In SPECT MPI, a technique for which we now have decades of data from clinical registries, dramatic declines in the frequency of abnormal studies have been reported over the last twenty years, from nearly $41 \%$ in 1991 to under $9 \%$ in 2009 in a single-center study of almost 40,000 patients ${ }^{44}$ (Fig 7). If broadly generalizable, the declining frequency and severity of myocardial ischemia may reflect a temporal change toward utilization of MPI for milder clinical presentations of CAD and underscore the need to refine future diagnostic workup of patients with suspected CAD in a cost-effective manner. ${ }^{45}$ Although a subset of these patients may not benefit from MPI at all (i.e., the patient without typical angina who can achieve a target heart rate on exercise treadmill testing), another subset of symptomatic patients who may not demonstrate ischemia associated with flow-limiting CAD may have diffuse atherosclerosis and microvascular dysfunction for which a more sensitive, quantitative assessment of myocardial blood flow and CFR, may diagnose abnormalities.

\section{Tailoring the imaging test to the clinical question}

From the discussion above, it is clear that no single imaging modality can provide all the answers in every patient. While some have advocated for the search of the imaging modality that can be used as the "one-stop shop", we prefer to think of the ideal modern CV imager as an expert with a bigger toolbox that provides complementary rather than competitive information. In some cases, as in patients on the very extreme spectrum of clinical risk, no imaging may be needed. As with anything in clinical medicine, Bayes theorem and pretest probabilities apply. The evolving clinical practice environment, which is moving from a focus on volume to value of diagnostic procedures, highlights the important role of the comprehensively trained cardiovascular consultant who has broad expertise across imaging modalities even while dedicated to one or two narrower fields.

It is likely that the role of radionuclide MPI will be reserved for the patient with intermediate-high likelihood of CAD, in who quantification of ischemia and risk stratification are key for management decisions, especially in the selection of patients for revascularization. Quantification of CFR as an integrator of the effect of stenosis severity, diffuse atherosclerosis, vessel remodeling, and microvascular dysfunction will likely enhance diagnostic and prognostic assessments in symptomatic patients with and without obstructive stenosis. This may add important information for guiding "personalized" therapy 
by identifying patients most likely to benefit from invasive procedures, as well as by identifying diffuse atherosclerosis and microvascular dysfunction as potentially relevant targets for aggressive therapeutic intervention and global CV risk reduction.

\section{Conclusion}

We are fully in an era of multimodality imaging in the CV realm. At their core, the multiple imaging techniques at our disposal share in common the need to be utilized judiciously in the context of patient-centered clinical care coupled with interdisciplinary clinical judgment, and to be constantly evaluated for their contributions to clinical outcomes.

\section{Abbreviations and Acronyms}

$\begin{array}{ll}\text { CAD } & \text { coronary artery disease } \\ \text { CCTA } & \text { coronary computed tomography angiography } \\ \text { CFR } & \text { coronary flow reserve } \\ \text { CMR } & \text { cardiac magnetic resonance imaging } \\ \text { CMRA } & \text { cardiac magnetic resonance imaging angiography } \\ \text { CTP } & \text { computed tomography perfusion } \\ \text { CV } & \text { cardiovascular } \\ \text { ECG } & \text { electrocardiogram } \\ \text { ETT } & \text { exercise treadmill testing } \\ \text { LV } & \text { left ventricular } \\ \text { MBF } & \text { myocardial blood flow } \\ \text { MI } & \text { myocardial infarction } \\ \text { MPI } & \text { myocardial perfusion imaging } \\ \text { PET } & \text { positron emission tomography } \\ \text { SE } & \text { stress echocardiography } \\ \text { SPECT } & \text { single photon emission tomography }\end{array}$

\section{References}

1. Beller GA, Zaret BL. Contributions of nuclear cardiology to diagnosis and prognosis of patients with coronary artery disease. Circulation. 2000; 101:1465-1478. [PubMed: 10736294]

2. Collins FS, Varmus H. A new initiative on precision medicine. N Engl J Med. 2015; 372:793-795. [PubMed: 25635347]

3. Shaw LJ, Marwick TH, Zoghbi WA, et al. Why all the focus on cardiac imaging? J Am Coll Cardiol Img. 2010; 3:789-794. 
4. Pennell DJ, Sechtem UP, Higgins CB, et al. Clinical indications for cardiovascular magnetic resonance (CMR): consensus panel report. J Cardiovasc Magn Reson. 2004; 6:727-765. [PubMed: 15646878]

5. Wolk MJ, Bailey SR, Doherty JU, et al. ACCF/AHA/ASE/ASNC/HFSA/HRS/SCAI/SCCT/ SCMR/STS 2013 multimodality appropriate use criteria for the detection and risk assessment of stable ischemic heart disease: a report of the American College of Cardiology Foundation appropriate use criteria task force, American Heart Association, American Society of Echocardiography, American Society of Nuclear Cardiology, Heart Failure Society of America, Heart Rhythm Society, Society for Cardiovascular Angiography and Interventions, Society of Cardiovascular Computed Tomography, Society for Cardiovascular Magnetic Resonance, and Society of Thoracic Surgeons. J Am Coll Cardiol. 2014; 63:380-406. [PubMed: 24355759]

6. Vesely MR, Dilsizian V. Nuclear cardiac stress testing in the era of molecular medicine. J Nucl Med. 2008; 49:399-413. [PubMed: 18322120]

7. Patterson RE, Horowitz SF, Eisner RL. Comparison of modalities to diagnose coronary artery disease. Semin Nucl Med. 1994; 24:286-310. [PubMed: 7817201]

8. Miller TD, Hodge DO, Christian TF, Milavetz JJ, Bailey KR, Gibbons RJ. Effects of adjustment for referral bias on the sensitivity and specificity of single photon emission computed tomography for the diagnosis of coronary artery disease. Am J Med. 2002; 112:290-297. [PubMed: 11893368]

9. Schuijf JD, Poldermans D, Shaw LJ, et al. Diagnostic and prognostic value of non-invasive imaging in known or suspected coronary artery disease. Eur J Nucl Med Mol Imaging. 2006; 33:93-104. [PubMed: 16320016]

10. Klocke FJ, Baird MG, Lorell BH, et al. ACC/AHA/ASNC guidelines for the clinical use of cardiac radionuclide imaging - executive summary: a report of the American College of Cardiology/ American Heart Association Task Force on Practice Guidelines (ACC/AHA/ASNC committee to revise the 1995 guidelines for the clinical use of cardiac radionuclide imaging). Circulation. 2003; 108:1404-1418. [PubMed: 12975245]

11. Di Carli MF, Dorbala S, Meserve J, El Fakhri G, Sitek A, Moore SC. Clinical myocardial perfusion PET/CT. J Nucl Med. 2007; 48:783-793. [PubMed: 17475968]

12. Parker MW, Iskandar A, Limone B, et al. Diagnostic accuracy of cardiac positron emission tomography versus single photon emission computed tomography for coronary artery disease: a bivariate meta-analysis. Circ Cardiovasc Imaging. 2012; 5:700-707. [PubMed: 23051888]

13. Mc Ardle BA, Dowsley TF, deKemp RA, Wells GA, Beanlands RS. Does rubidium-82 PET have superior accuracy to SPECT perfusion imaging for the diagnosis of obstructive coronary disease? A systematic review and meta-analysis. J Am Coll Cardiol. 2012; 60:1828-1837. [PubMed: 23040573]

14. Dorbala S, Di Carli MF. Cardiac PET perfusion: prognosis, risk stratification, and clinical management. Semin Nucl Med. 2014; 44:344-357. [PubMed: 25234079]

15. Nesto RW, Kowalchuk GJ. The ischemic cascade: temporal sequence of hemodynamic, electrocardiographic and symptomatic expressions of ischemia. Am J Cardiol. 1987; 59:23C-30C.

16. Schinkel AF, Bax JJ, Geleijnse ML, et al. Noninvasive evaluation of ischaemic heart disease: myocardial perfusion imaging or stress echocardiography? Eur Heart J. 2003; 24:789-800. [PubMed: 12727146]

17. Gebker R, Jahnke C, Manka R, et al. Additional value of myocardial perfusion imaging during dobutamine stress magnetic resonance for the assessment of coronary artery disease. Circ Cardiovasc Imaging. 2008; 1:122-130. [PubMed: 19808529]

18. Min JK, Shaw LJ. Noninvasive diagnostic and prognostic assessment of individuals with suspected coronary artery disease: coronary computed tomographic angiography perspective. Circ Cardiovasc Imaging. 2008; 1:270-281. [discussion 281]. [PubMed: 19808551]

19. Budoff MJ, Dowe D, Jollis JG, et al. Diagnostic performance of 64-multidetector row coronary computed tomographic angiography for evaluation of coronary artery stenosis in individuals without known coronary artery disease: results from the prospective multicenter accuracy (assessment by coronary computed tomographic angiography of individuals undergoing invasive coronary angiography) trial. J Am Coll Cardiol. 2008; 52:1724-1732. [PubMed: 19007693] 
20. Di Carli MF, Dorbala S, Curillova Z, et al. Relationship between CT coronary angiography and stress perfusion imaging in patients with suspected ischemic heart disease assessed by integrated PET-CT imaging. J Nucl Cardiol. 2007; 14:799-809. [PubMed: 18022106]

21. Schuijf JD, Wijns W, Jukema JW, et al. Relationship between noninvasive coronary angiography with multi-slice computed tomography and myocardial perfusion imaging. J Am Coll Cardiol. 2006; 48:2508-2514. [PubMed: 17174190]

22. Iskandrian AS, Chae SC, Heo J, Stanberry CD, Wasserleben V, Cave V. Independent and incremental prognostic value of exercise single-photon emission computed tomographic (SPECT) thallium imaging in coronary artery disease. J Am Coll Cardiol. 1993; 22:665-670. [PubMed: 8354796]

23. Hachamovitch R, Berman DS, Shaw LJ, et al. Incremental prognostic value of myocardial perfusion single photon emission computed tomography for the prediction of cardiac death: differential stratification for risk of cardiac death and myocardial infarction. Circulation. 1998; 97:535-543. [PubMed: 9494023]

24. Hachamovitch R, Berman DS, Kiat H, et al. Exercise myocardial perfusion SPECT in patients without known coronary artery disease: incremental prognostic value and use in risk stratification. Circulation. 1996; 93:905-914. [PubMed: 8598081]

25. Shaw LJ, Iskandrian AE. Prognostic value of gated myocardial perfusion SPECT. J Nucl Cardiol. 2004; 11:171-185. [PubMed: 15052249]

26. Sharir T, Germano G, Kang X, et al. Prediction of myocardial infarction versus cardiac death by gated myocardial perfusion SPECT: risk stratification by the amount of stress-induced ischemia and the poststress ejection fraction. J Nucl Med. 2001; 42:831-837. [PubMed: 11390544]

27. Gould KL. Does coronary flow trump coronary anatomy? JACC Cardiovasc Imaging. 2009; 2:1009-1023. [PubMed: 19679290]

28. El Fakhri G, Kardan A, Sitek A, et al. Reproducibility and accuracy of quantitative myocardial blood flow assessment with (82)rb PET: comparison with (13)n-ammonia PET. J Nucl Med. 2009; 50:1062-1071. [PubMed: 19525467]

29. El Fakhri G, Sitek A, Guerin B, Kijewski MF, Di Carli MF, Moore SC. Quantitative dynamic cardiac 82rb PET using generalized factor and compartment analyses. J Nucl Med. 2005; 46:1264-1271. [PubMed: 16085581]

30. Murthy VL, Naya M, Foster CR, et al. Improved cardiac risk assessment with noninvasive measures of coronary flow reserve. Circulation. 2011; 124:2215-2224. [PubMed: 22007073]

31. Ziadi MC, Dekemp RA, Williams KA, et al. Impaired myocardial flow reserve on rubidium- 82 positron emission tomography imaging predicts adverse outcomes in patients assessed for myocardial ischemia. J Am Coll Cardiol. 2011; 58:740-748. [PubMed: 21816311]

32. Fukushima K, Javadi MS, Higuchi T, et al. Prediction of short-term cardiovascular events using quantification of global myocardial flow reserve in patients referred for clinical 82rb PET perfusion imaging. J Nucl Med. 2011; 52:726-732. [PubMed: 21498538]

33. Herzog BA, Husmann L, Valenta I, et al. Long-term prognostic value of 13n-ammonia myocardial perfusion positron emission tomography added value of coronary flow reserve. J Am Coll Cardiol. 2009; 54:150-156. [PubMed: 19573732]

34. Naya M, Murthy VL, Taqueti VR, et al. Preserved coronary flow reserve effectively excludes highrisk coronary artery disease on angiography. J Nucl Med. 2014; 55:248-255. [PubMed: 24408896]

35. Taqueti VR, Hachamovitch R, Murthy VL, et al. Global coronary flow reserve is associated with adverse cardiovascular events independently of luminal angiographic severity and modifies the effect of early revascularization. Circulation. 2015; 131:19-27. [PubMed: 25400060]

36. Nahser PJ Jr, Brown RE, Oskarsson H, Winniford MD, Rossen JD. Maximal coronary flow reserve and metabolic coronary vasodilation in patients with diabetes mellitus. Circulation. 1995; 91:635640. [PubMed: 7828287]

37. Murthy VL, Naya M, Foster CR, et al. Association between coronary vascular dysfunction and cardiac mortality in patients with and without diabetes mellitus. Circulation. 2012; 126:18581868. [PubMed: 22919001] 
38. Taqueti VR, Everett BM, Murthy VL, et al. Interaction of impaired coronary flow reserve and cardiomyocyte injury on adverse cardiovascular outcomes in patients without overt coronary artery disease. Circulation. 2015; 131:528-535. [PubMed: 25480813]

39. Bittencourt MS, Hulten E, Ghoshhajra B, et al. Prognostic value of nonobstructive and obstructive coronary artery disease detected by coronary computed tomography angiography to identify cardiovascular events. Circ Cardiovasc Imaging. 2014; 7:282-291. [PubMed: 24550435]

40. Hachamovitch R, Nutter B, Hlatky MA, et al. Patient management after noninvasive cardiac imaging results from SPARC (study of myocardial perfusion and coronary anatomy imaging roles in coronary artery disease). J Am Coll Cardiol. 2012; 59:462-474. [PubMed: 22281249]

41. Gowd BM, Heller GV, Parker MW. Stress-only SPECT myocardial perfusion imaging: a review. J Nucl Cardiol. 2014; 21:1200-1212. [PubMed: 25005348]

42. Berman DS, Maddahi J, Tamarappoo BK, et al. Phase ii safety and clinical comparison with singlephoton emission computed tomography myocardial perfusion imaging for detection of coronary artery disease: flurpiridaz F 18 positron emission tomography. J Am Coll Cardiol. 2013; 61:469_ 477. [PubMed: 23265345]

43. Yu M, Guaraldi MT, Mistry M, et al. BMS-747158-02: a novel PET myocardial perfusion imaging agent. J Nucl Cardiol. 2007; 14:789-798. [PubMed: 18022105]

44. Rozanski A, Gransar H, Hayes SW, et al. Temporal trends in the frequency of inducible myocardial ischemia during cardiac stress testing: 1991 to 2009. J Am Coll Cardiol. 2013; 61:1054-1065. [PubMed: 23473411]

45. Iskandrian AE, Hage FG. Declining frequency of ischemia detection using stress myocardial perfusion imaging. J Am Coll Cardiol. 2013; 61:1066-1068. [PubMed: 23473412] 


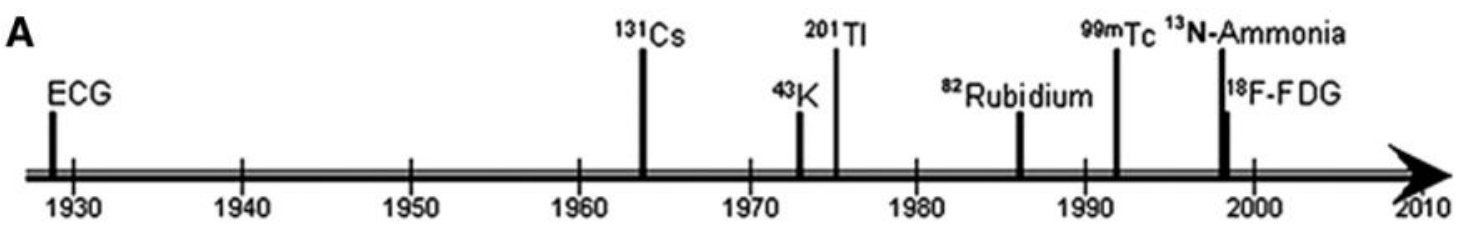

B

\section{ECG}
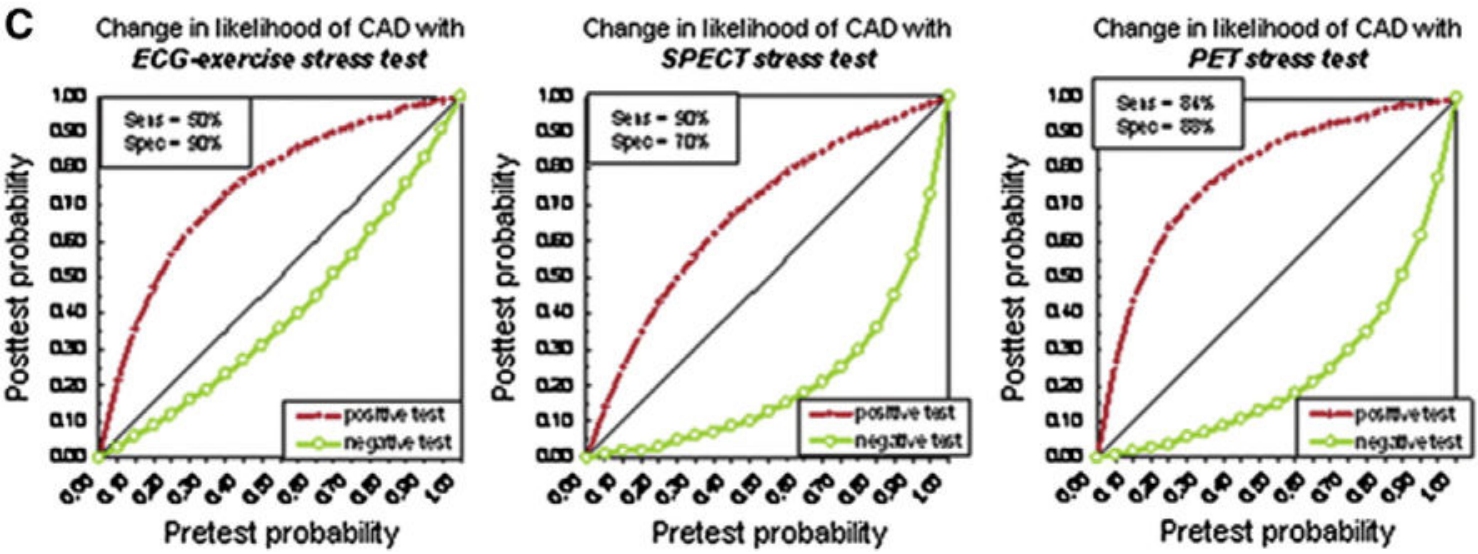

Fig 1.

Timeline of signal and detector advances in the history of radionuclide myocardial perfusion imaging and accuracy of current techniques. (A) Time points of initial recognition that the electrocardiogram (ECG) and radiotracers provide diagnostic information for myocardial perfusion and metabolism. (B) Time span of use of detection modalities in cardiac stress testing. (C) Diagnostic information provided by stress testing as change from pretest to posttest likelihood of angiographically confirmed CAD. Reproduced with permission from the Journal of Nuclear Medicine, () by the Society of Nuclear Medicine and Molecular Imaging. 6 

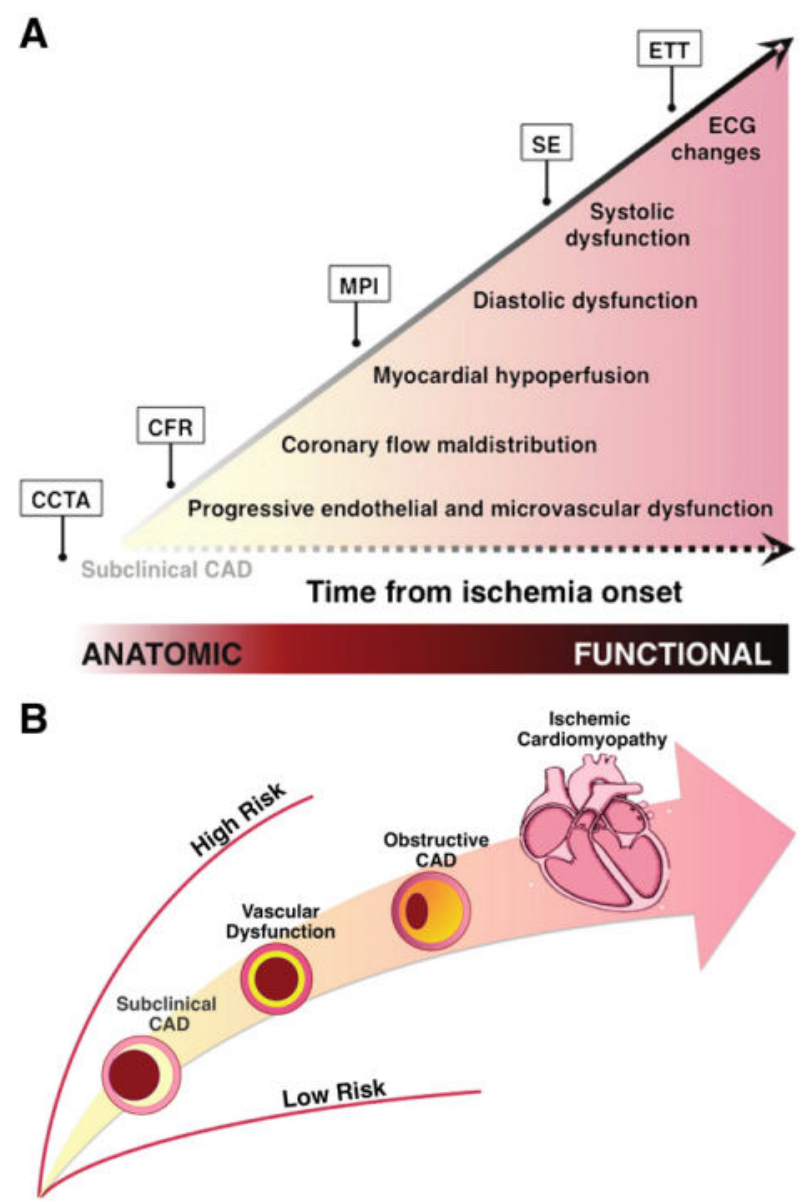

Fig 2.

Multimodality cardiovascular imaging for the evaluation of patients with known or suspected coronary artery disease (CAD). (A) Schematic of the ischemic cascade, a sequence of pathophysiologic events associated with $\mathrm{CAD}$. Anatomy-based imaging probes earlier events in the cascade than does perfusion-based imaging. (B), Spectrum of CAD in ischemic heart disease. Abbreviations: CCTA, coronary computed tomography angiography; CFR, coronary flow reserve; MPI, myocardial perfusion imaging; SE, stress echocardiography; ETT, exercise treadmill testing. 


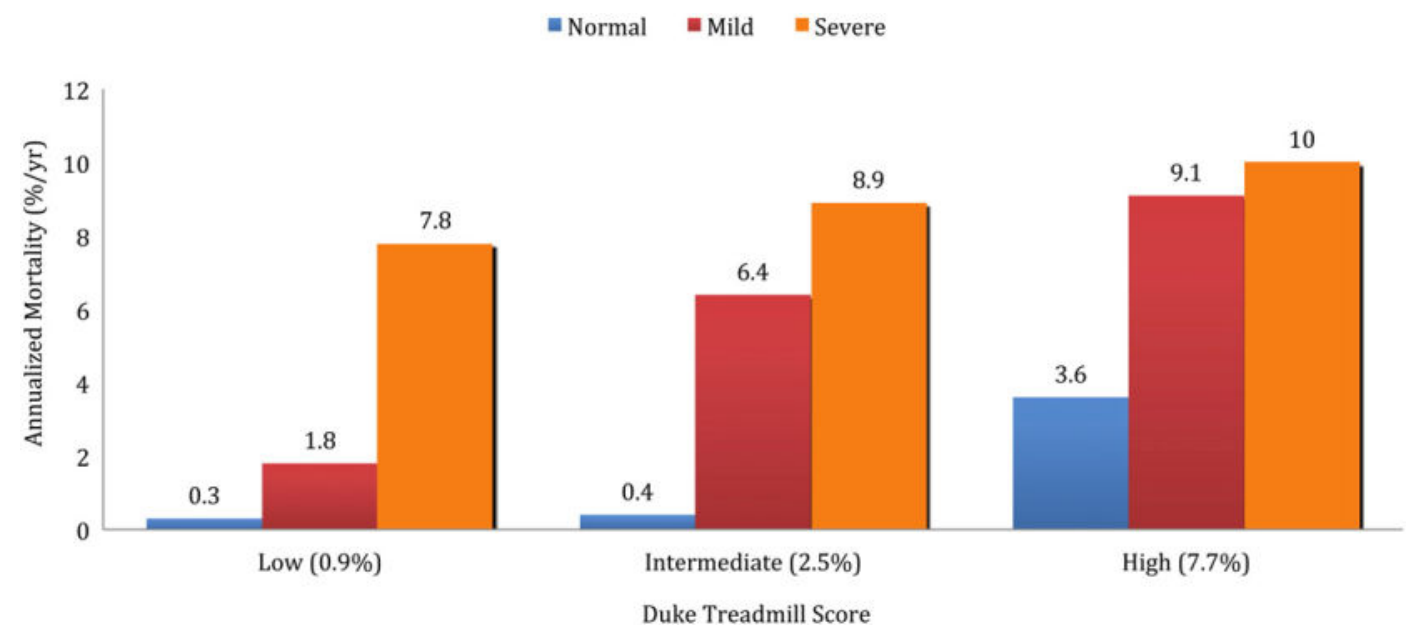

Fig 3.

Rates of cardiac death and myocardial infarction in patients without prior CAD undergoing stress SPECT. Patients are separated into low, intermediate and high Duke treadmill scores. Patients are further stratified by stress SPECT results (normal, mildly and moderately to severely abnormal scans). A significant difference in event rates across scan categories ( $p<$ $0.05)$ is present in the Duke treadmill score subgroups as a function of scan result. Data obtained from Hachamovitch et al. ${ }^{24}$ 


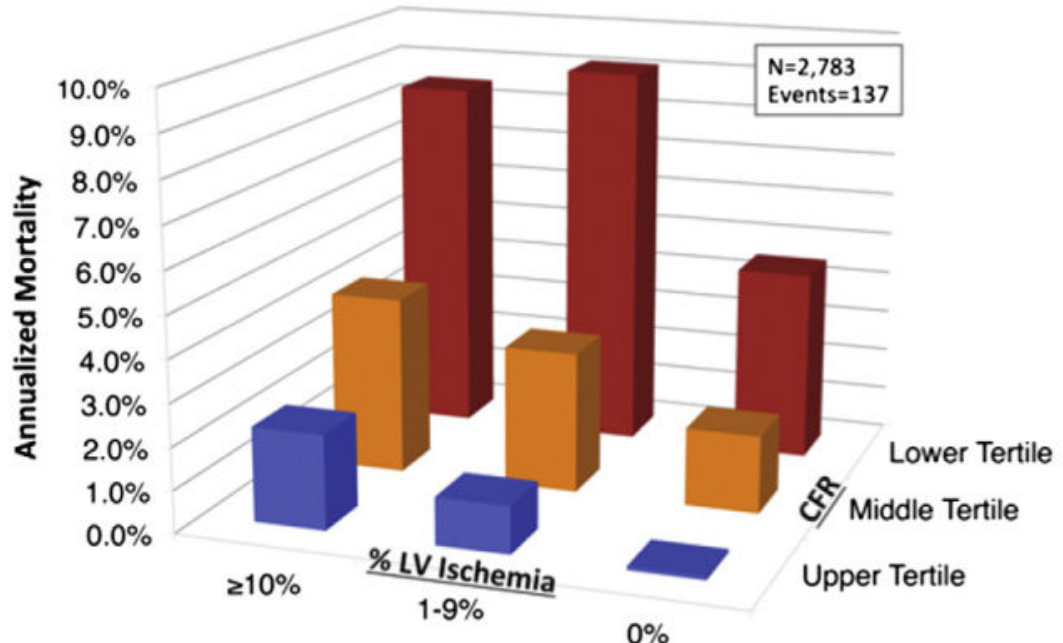

\begin{tabular}{|l|l|l|c|}
\cline { 2 - 4 } \multicolumn{1}{c|}{} & $210 \%$ & $1-9 \%$ & $0 \%$ \\
\hline "Upper Tertile & $2.2 \%$ & $1.1 \%$ & $0.1 \%$ \\
\hline "Middle Tertile & $4.2 \%$ & $3.3 \%$ & $1.8 \%$ \\
\hline "Lower Tertile & $8.5 \%$ & $9.1 \%$ & $4.5 \%$ \\
\hline
\end{tabular}

Fig 4.

Unadjusted annualized cardiac mortality by tertiles of coronary flow reserve (CFR) and categories of left ventricular myocardial ischemia (\% LV ischemia). Lower CFR consistently identified higher-risk patients at every level of myocardial ischemia, including among those with visually normal PET scans. Adapted with permission. ${ }^{30}$ 
A) Unadjusted

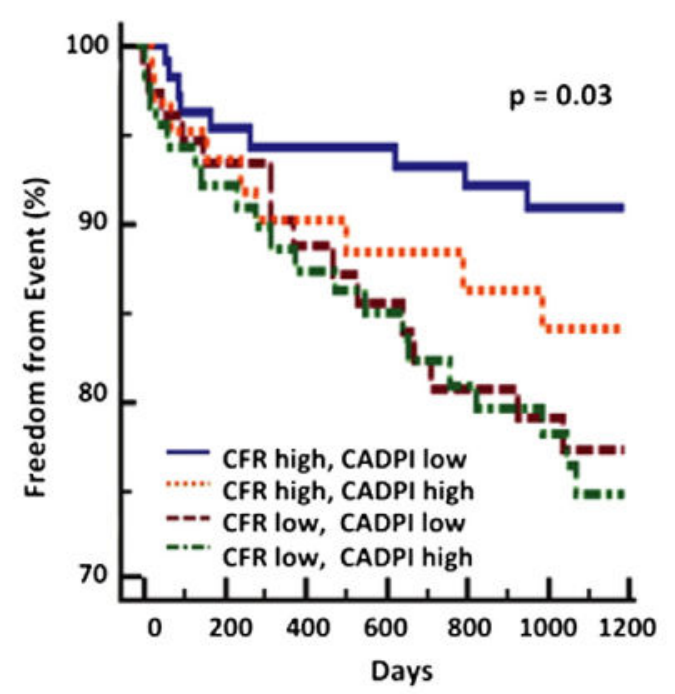

B) Adjusted ${ }^{\text {t }}$

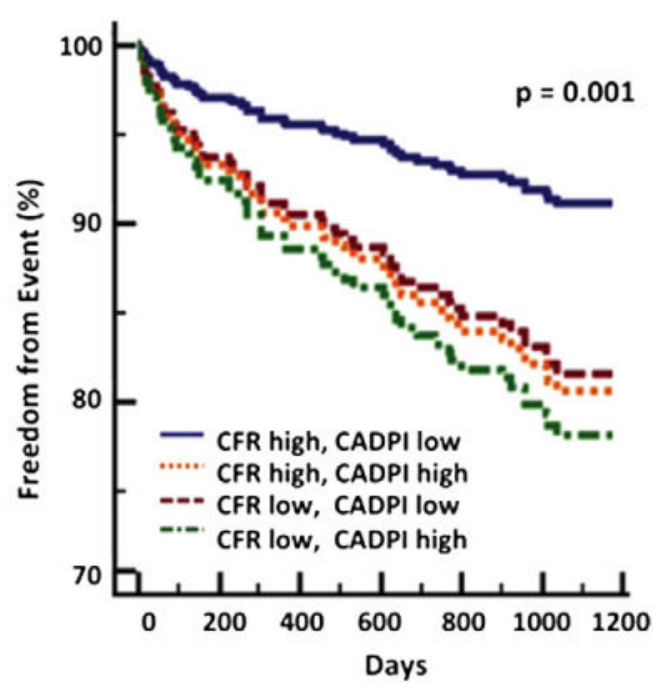

\begin{tabular}{lccc}
\hline & Total (N = 329) & Early Revascularization (\%) & by PCI (\%) \\
\hline CFR high, CADPI low & 106 & $38(35.8)$ & $34(89.5)$ \\
CFR high, CADPI high & 60 & $49(81.7)$ & $36(73.5)$ \\
CFR low, CADPI low & 74 & $35(47.3)$ & $34(97.1)$ \\
CFR low, CADPI high & 89 & $71(79.8)$ & $50(70.4)$ \\
\hline
\end{tabular}

'CFR denotes coronay flow reserve: high ( 21.6$)$, low $(<1.6)$.

'CADPI denotes coronary artery disease prognostic index: low (<37), high ( 237 , reflects $>70 \%$ stenosis in $>1$ epicardial artery). 'Adjusted for pretest clinical score, left ventricular ejection fraction, left ventricular ischemia, and early revascularization with percutaneous coronary intervention ( $\mathrm{PCI})$ or coronary artery bypass grafting (CABG).

Early Revascularization denotes that within 90 days of noninvasive imaging.

Fig 5.

Freedom from cardiovascular death or heart failure admission according to coronary flow reserve (CFR) and invasive luminal angiographic score (CAD prognostic index, CADPI).

(A) Freedom from events differed significantly among subgroups stratified by CFR and CADPI, such that patients with low CFR, independently of angiographic disease score, experienced higher rates of events (overall $p=0.03$ ). (B) In adjusted analysis, patients with low CFR experienced rates of events similar to those of patients with high CADPI. PCI, percutaneous coronary intervention. Reproduced with permission. ${ }^{35}$ 


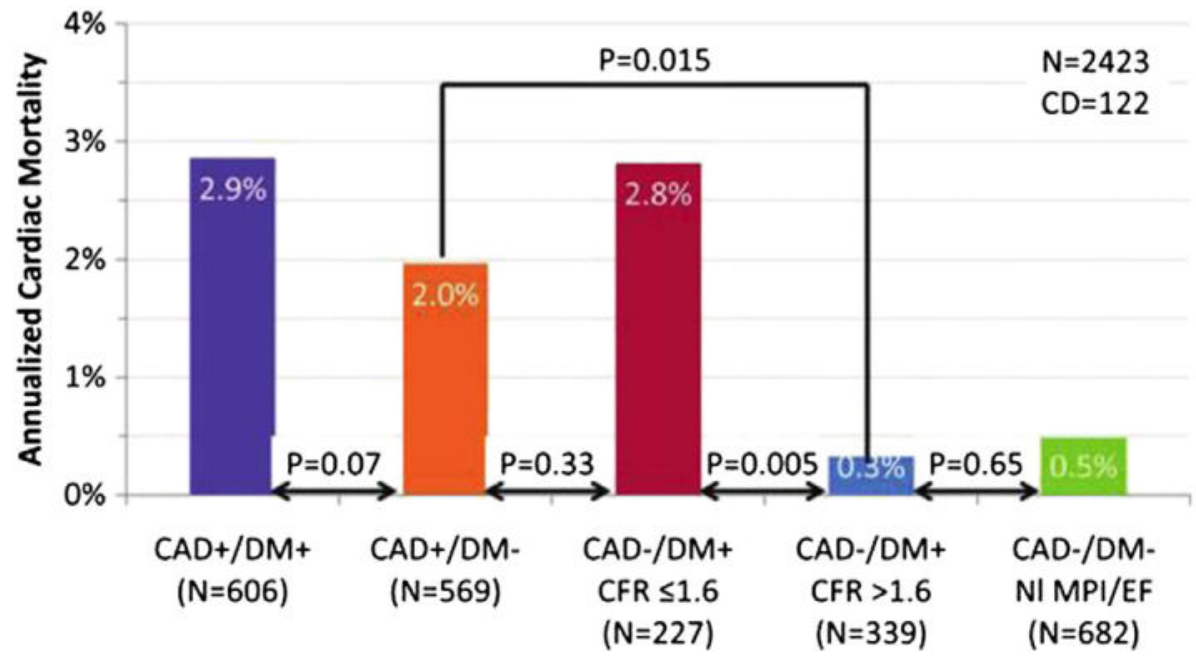

Fig 6.

Adjusted annualized cardiac mortality among patients with diabetes mellitus (DM) and/or CAD (i.e., history of coronary revascularization or myocardial infarction) by coronary flow reserve (CFR). NI MPI/EF indicated normal perfusion imaging/ejection fraction. Adapted with permission. ${ }^{37}$ 
abnormal perfusion (\%) @ Annual mortality (\%)

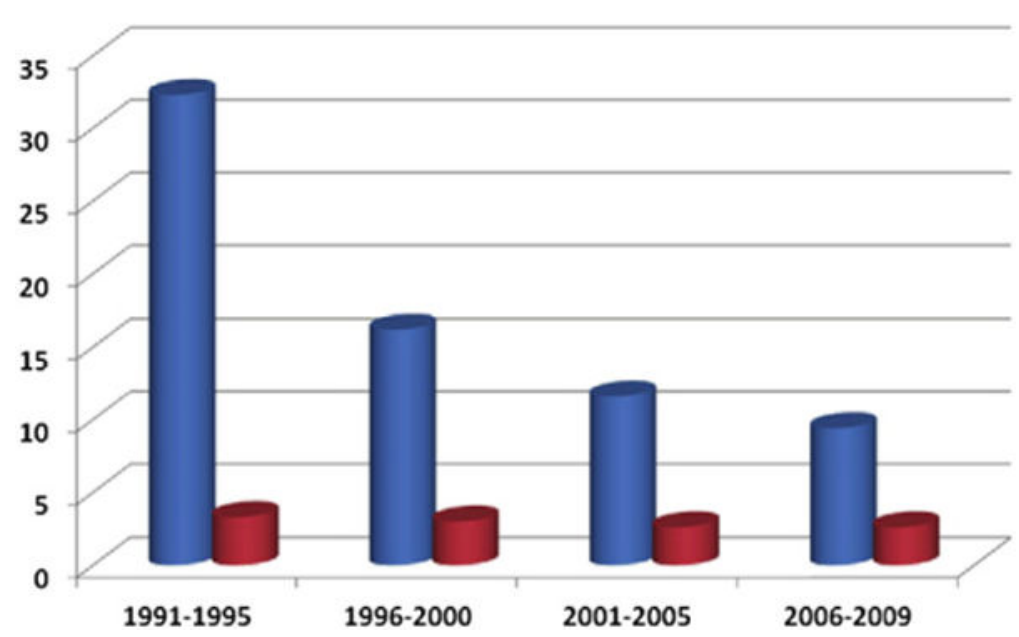

Fig 7.

The frequency of abnormal perfusion (defined as summed stress score involving more than $5 \%$ of the left ventricle) and annual mortality rates on stress myocardial perfusion imaging shown for four successive time periods. Reproduced with permission. ${ }^{45}$ 